\title{
Recursos educacionais abertos (REA) e novas práticas sociais
}

\section{Open educational resources (OER) and new social practices}

\section{Recurso educativos abiertos (REA) y nuevas prácticas sociales}

\author{
Priscila Gonsales | prigon@educadigital.org.br \\ Instituto Educadigital, Diretoria Educadigital. São Paulo, SP, Brasil.
}

\section{Resumo}

Segundo definição da Unesco e da Commonwelth of Learning, recursos educacionais abertos (REA) são materiais de ensino, aprendizado e pesquisa, disponíveis em qualquer suporte ou mídia, preferencialmente em plataformas ou formatos livres (software livre), que estejam sob domínio público ou licenciados de maneira aberta, permitindo que sejam utilizados ou adaptados por terceiros. No contexto da cultura digital, os REA criam a oportunidade para que educadores, estudantes e mesmo aqueles não estejam formalmente vinculados a uma instituição de ensino se envolvam no processo criativo de desenvolver e adaptar recursos educacionais. Governos e instituições de ensino podem formar professores e alunos para a produção colaborativa de textos, imagens e vídeos de qualidade. Com a abertura dos materiais na internet, a possibilidade de formação continuada se expande a toda a sociedade.

Palavras-chave: Educação; Educação continuada; Ensino; Cultura digital; Direito autoral; Material didático.

\begin{abstract}
According to UNESCO and Commonwealth of Learning, open educational resources (OER) are teaching, learning and research materials in any medium, digital or otherwise, that are in the public domain or have been released under an open license that permits free access, use, adaptation and redistribution by others. In the context of digital culture, OER create the opportunity for educators, students and even those are not formally tied to an educational institution to be involved in the creative process to develop and adapt educational resources. Governments and educational institutions can train teachers and students for collaborative production of texts, pictures and quality videos. With the opening of the materials on internet, the possibility of continuing education extends to the whole society.
\end{abstract}

Keywords: Education; Education continuing; Teaching; Digital culture; Author rights; Textbooks. 


\section{Resumen}

Según la definición de la UNESCO y del Commonwealth of Learning, recursos educativos abiertos (REA) son materiales de enseñanza, aprendizaje, y investigación disponibles en cualquier medio o soporte, de preferencia en las plataformas o formatos abiertos (software libre), que están en el dominio público o bajo licencias abiertas, lo que les permite ser utilizados o adaptados por otros. En el contexto de la cultura digital, REA crea la oportunidad para que los educadores, estudiantes e incluso aquellos que no están vinculados formalmente a una institución educativa participen del proceso creativo para desarrollar y adaptar los recursos educativos. Los gobiernos y las instituciones educativas pueden formar profesores y estudiantes para la producción colaborativa de textos, fotos y videos de calidad. Con la apertura de los materiales en internet, la posibilidad de la educación continuada se extiende a toda la sociedad.

Palabras clave: Educación; Educación continuada; Enseñanza; Cultura digital; Derecho autoral; Material didáctico.

Declaração de conflito de interesses: Não há conflito de interesses.

Agradecimento/Contribuições adicionais: Debora Sebriam e Carolina Rossini pela parceria de trabalho, advocacy e pesquisa para a causa REA no Brasil.

Histórico do artigo: Submetido: 26.jan.2016 | Aceito: 27.jan.2016 | Publicado: 31.mar.2016

Licença: CC BY-NC atribuição não comercial. Com essa licença é permitido acessar, baixar (download), copiar, imprimir, compartilhar, reutilizar e distribuir os artigos, desde que para uso não comercial e com a citação da fonte, conferindo os devidos créditos de autoria e menção à Reciis. Nesses casos, nenhuma permissão é necessária por parte dos autores ou dos editores 
Lançada em setembro de 2015, a sexta edição da pesquisa TIC (Tecnologias de Informação e Comunicação) Educação ${ }^{1}$, que analisa o uso das tecnologias digitais, traz pelo terceiro ano consecutivo dados referentes ao uso, pelos educadores, de objetos digitais de aprendizagem disponíveis online. Os números são espantosos: 96\% dos professores afirmam que preparam suas aulas com materiais encontrados na internet, sendo que 92\% afirmaram que fazem isso por motivação própria. No entanto, mais da metade deles gostaria de conhecer metodologias inovadoras para uso de tecnologias digitais com os alunos.

A escola que temos hoje ainda segue marcada pela dinâmica da sociedade industrial, de "produção" em classes seriadas, com uso de materiais fechados (copyright), proprietários e sem possibilidade de adaptação, em que todos devem aprender o mesmo conteúdo, da mesma forma e ao mesmo tempo. Sem falar da estrutura de salas de aula onde ainda predomina a comunicação de um (no caso o professor) para muitos (os alunos), ou seja, centralizada, unidirecional. Mesmo com décadas de estudos e pesquisas na área de pedagogia, somados ao trabalho de mentores do porte de Paulo Freire e Anísio Teixeira ${ }^{\text {ii }}$ - para ficar só nos brasileiros - apontando que esse formato não corresponde ao que se espera de uma educação de qualidade, foi preciso que chegasse a era digital para esse questionamento de fato acontecer - ou melhor - ser levado a sério.

Era digital, sociedade informacional, sociedade em rede são algumas das terminologias mais utilizadas por estudiosos contemporâneos como Manuel Castells ${ }^{\text {iiii }}$, Pierre Levy ${ }^{\mathrm{iv}}$, André Lemos ${ }^{\mathrm{v}}$, chamando a atenção para o momento revolucionário em que estamos vivendo, no qual a colaboração e o compartilhamento vão ganhando força nas relações entre as pessoas, seja no ambiente pessoal, profissional ou acadêmico. $O$ ato de possuir algo vem perdendo espaço para a necessidade de ter acesso a algo; a oferta de serviços customizados cresce em relação à de produtos prontos. E isso tem tudo ver com educação aberta e práticas educativas abertas.

Trata-se de uma mudança drástica e necessária. Criado na década de 1930, por um decreto-lei nacional, o livro didático ${ }^{3}$ faz parte da memória de muitas gerações em sua vida escolar, por ser o grande responsável pela organização do conteúdo estudado, por ter um projeto visual para chamar a atenção, para estimular a concentração e o estudo, por apresentar também pontos do cenário político e econômico do país. O livro didático teve e ainda tem um papel fundamental na democratização do acesso à educação, e o atual programa oficial vigente (Programa Nacional do Livro Didático - PNLD) tem muito mérito por permitir que os educadores decidam quais livros usar depois de avaliação prévia do Ministério da Educação.

No entanto, no contexto multimídia do século XXI, o livro didático fica restrito a uma concepção linear e a uma lógica top-down (de cima para baixo) que já não é mais coerente com as formas de ser e estar em sociedade. É preciso deixar de lado o foco no consumo de informações para priorizar a produção de informação, conhecimento e cultura por professores e alunos nas escolas. Qualquer dispositivo conectado à internet possibilita hoje a entrada em museus do mundo todo, o acompanhamento de aulas e cursos online, a pesquisa de estudos e teses e, especialmente, participar de redes online, usar materiais nos mais diversos formatos, enfim, uma infinidade de possibilidades. Mas, se esse uso continuar pautado pela lógica do consumo, pouco vamos avançar em termos de educar no contexto da cultura digital.

A pesquisa acima citada mostra que os próprios professores querem produzir suas aulas com recursos digitais variados que encontram na internet e não mais simplesmente "seguir" o livro. Porém, a imensa maioria dos materiais e recursos disponíveis na web são gratuitos, mas pautados pela lógica do consumo. Deveriam, sobretudo os financiados com recursos públicos, ser abertos, invertendo essa lógica para um novo modelo mental, o maker, de produção pelos usuários. E a diferença é muito grande. Se um professor deseja usar parte de um vídeo encontrado em repositórios como o Youtube, por exemplo, para elaborar um

http://www.paulofreire.org/paulo-freire-patrono-da-educacao-brasileira

http://educadores.educacao.ba.gov.br/patrono-anisio-teixeira

http://www.manuelcastells.info/en

http://pierrelevyblog.com

http://buscatextual.cnpq.br/buscatextual/visualizacv.do?metodo=apresentar\&id=K4780048D9 
outro vídeo dentro de uma proposta pedagógica, isto é, produzir e não apenas consumir, ele não vai poder fazer isso hoje. Não sem a expressa autorização do autor daquele vídeo. O curioso é que, muitas vezes, o autor do vídeo não se importaria, ou até ficaria muito orgulhoso de ver usa obra inspirando outras, mas ele não sabe que pode deixar essa opção visível para o usuário a partir da escolha de uma licença flexível.

Ao falarmos de licença flexível, chegamos ao conceito de REA, ou recursos educacionais abertos, e sua importância no contexto da cultura digital em que estamos. A expressão "recursos educacionais abertos" (open educational resources, em inglês OER) foi adotada, pela primeira vez, durante um fórum da Unesco em 2002. Trata-se do esforço de uma comunidade global articulada de educadores, políticos e usuários para criar, reutilizar e propagar bens educacionais pertencentes à humanidade, bens esses cada vez mais acessíveis graças à internet.

Anualmente, uma quantidade imensa de dinheiro público (da ordem de milhões) é gasta pelos governos na compra de materiais didáticos impressos e digitais que não são REA e, portanto, são de acesso restrito, inibindo as possibilidades de reprodução, criação e adaptação de conteúdos por educadores e estudantes. Em 2012, a Unesco realizou em Paris o Congresso Mundial de REA, que gerou uma declaração ${ }^{6}$ convidando governos do mundo todo a determinar que recursos educacionais financiados com recursos públicos devem adotar o modelo REA.

Por definição oficial divulgada em documento da Unesco/Commonwealth7 ${ }^{7}$ REA são materiais de ensino, aprendizado e pesquisa, fixados em qualquer suporte ou mídia, preferencialmente em plataformas ou formatos livres (software livre), que estejam sob domínio público ou licenciados de maneira aberta, permitindo que sejam utilizados ou adaptados por terceiros. Recursos educacionais abertos podem incluir cursos completos, partes de cursos, módulos, livros didáticos, artigos de pesquisa, vídeos, testes, software, e qualquer outra ferramenta, material ou técnica que possa apoiar o acesso ao conhecimento. Tal definição data de 2011 e foi redigida com ajuda da Comunidade Recursos Educacionais Abertos (REA) do Brasil.

A lei de direito autoral brasileira ainda não foi reformulada para atender à demanda das novas práticas sociais que estão surgindo, especialmente a de criação e adaptação de materiais audiovisuais os mais diversos. Segundo a lei, o autor (ou o detentor dos direitos do autor) é o único proprietário dos direitos de uso de sua obra, cabendo a ele decidir quando e como permite o uso por terceiros. Não existe exceção nem para finalidade educativa, e a possibilidade de uso de pequenos trechos é bastante controversa. Com isso, é possível afirmar que os educadores estariam sujeitos a punições.

Mesmo que a obra não traga o símbolo (c) de "copyright" ou a frase "todos os direitos reservados", a lei garante que a obra é copyright. Mesmo citando a fonte, se não há uma licença indicada nada se pode fazer além de olhar, ler ou apreciar a obra somente no formato em que ela se encontra disponível. Como essa questão de direito autoral não se restringe ao Brasil, em meados dos anos 2000, surge nos EUA uma ONG chamada Creative Commons que começa a disseminar em todo o mundo um modelo de gestão de direitos autorais em que o autor pode optar por uma licença mais flexível, concedendo de forma clara alguns direitos de uso de sua obra. Qualquer pessoa interessada em licenciar sua obra de forma aberta pode acessar o site do $\mathrm{CC}$, responder a algumas perguntas, e instantaneamente, receber uma das seis licenças disponíveis para deixar ou incorporar em sua obra.

Os REA criam a oportunidade para uma transformação fundamental na educação: a autoria. Permitem que educadores, estudantes e mesmo aqueles não estejam formalmente vinculados a uma instituição de ensino se envolvam no processo criativo de desenvolver e adaptar recursos educacionais. Governos e instituições de ensino podem formar professores e alunos para a produção colaborativa de textos, imagens e vídeos de qualidade. Com a abertura dos materiais na internet, a possibilidade de formação continuada se expande a toda a sociedade. 


\section{REA e a política pública}

Em abril de 2012, a Comunidade REA Brasil ${ }^{5}$, formada por pessoas de diversas áreas do saber, lançava na Casa da Cultura Digital ${ }^{\text {vi }}$, em São Paulo, o primeiro livro no país com artigos acadêmicos e relatos de experiências sobre REA na área de política pública e de práticas educativas.

Atuando junto à Comunidade REA desde 2008, o projeto REA.br² ${ }^{2}$, conduzido atualmente pelo Instituto Educadigital ${ }^{\text {vii }}$ com apoio financeiro das Open Society Foundations ${ }^{\text {viii }}$, vem trabalhando para transformar a política pública de acesso a recursos educacionais financiados com orçamento público. Alguns resultados já podem ser observados nos últimos três anos, tanto na esfera federal como estadual e municipal. Um deles é o Plano Nacional de Educação (PNE)4, aprovado em 2014, que contempla o incentivo aos REA dentro de duas de suas metas. Ainda no âmbito federal, o Projeto de Lei $1513 / 2011^{\text {ix }}$ visa garantir que as compras públicas ou contratação de serviços e materiais educacionais sejam regidas por meio de licenças livres, permitindo a difusão e a ampliação do acesso a esses bens por toda a sociedade.

Na esfera municipal, já existe uma política pública de REA vigente no município de São Paulo, desde o Decreto $52.681^{8}$, de 26 de setembro de 2011, que dispõe sobre o licenciamento obrigatório das obras intelectuais produzidas ou subsidiadas com objetivos educacionais, pedagógicos e afins, no âmbito da rede pública municipal de ensino. Hoje, quem entra no site ${ }^{x}$ da Secretaria Municipal de Educação de São Paulo já localiza a licença definida para uso dos materiais disponíveis.

Em agosto de 2015, o REA.br articulou com as comissões de Educação e Cultura da Câmara dos Deputados a organização de um Seminário Internacional sobre Recursos Internacionais Abertos ${ }^{\mathrm{xi}}$ em Brasília, voltada a pesquisadores e, principalmente, gestores e legisladores públicos. O evento foi realizado no auditório Nereu Ramos e contou com a presença de palestrantes internacionais, como Hal Plotkin, responsável pela implementação da política de REA do governo Obama nos EUA ${ }^{\text {xii. }}$

\section{Educação aberta como tendência}

Conteúdo aberto também já aparece como uma das tendências para a educação em um dos principais relatórios mundiais sobre o uso da tecnologia na educação, o Horizon Report ${ }^{\text {xiii }}$. Segundo o documento, a "educação aberta" e os "cursos abertos e gratuitos, como os Moocs9", surgem como forma de diminuir as barreiras de acesso à informação até para quem está fora da escola.

Na perspectiva apontada pelo Horizon Report, alguns projetos educacionais mais recentes já estão sendo criados dentro da perspectiva de REA, ressaltando não apenas a importância do bem público e da livre adaptação, como também a autoria dos educadores envolvidos.

A Secretaria Estadual de Educação de São Paulo lançou no início de 2014 um remix do site Escola Digital $^{\mathrm{kiv}}$, chamado Currículo Mais ${ }^{10}$, em que os próprios gestores e professores da rede são convidados a analisar e sugerir objetos digitais para os colegas.

O contexto atual, não só no Brasil, mas em todo o mundo, envolve o desafio de integrar - ou mais efetivamente, impregnar - as TIC ao currículo de forma qualitativa e trazer de fato a cultura digital para a escola e demais espaços de aprendizagem (sejam eles formais ou informais). Desafio esse que passa, em primeiro lugar, pela formação inicial e continuada de docentes e, simultaneamente, pela incorporação

vi Casa da Cultura Digital. Casa da Cultura Digital. [citado 27 jan. 2016]. Disponível em: www.casadaculturadigital.com.br

vii Instituto Educadigital. Apresentação. [citado 27 jan. 2016]. Disponível em: www.educadigital.org.br

viii http://www.opensocietyfoundations.org/

ix http://bit.ly/1i42Kba

x http://portalsme.prefeitura.sp.gov.br/default.aspx

xi http://www.rea.net.br/site/seminario-internacional-rea-na-integra/

xii http://www.ebc.com.br/recursos-educacionais-abertos

xiii $\quad$ http://migre.me/dl40j

xiv www.escoladigital.org.br 
de tendências que já fazem parte do cotidiano da sociedade conectada, tais como: personalização de uso, práticas colaborativas em redes digitais, adoção crescente de celulares e computadores móveis, e preferência por softwares livres e conteúdo aberto.

A educação precisa de apoio, mas esse apoio não pode vir de fora para dentro, justamente porque não existe receita pronta e única. É preciso estimular que professores sejam autores de seu próprio processo de formação, procurando não só usar REA mas também produzir e compartilhar suas produções, seus projetos pedagógicos, suas sequências didáticas, possibilitando que outros educadores possam aproveitar e remixar essas iniciativas de acordo com as características culturais de cada região.

Quando materiais didáticos e educacionais são considerados bens públicos e comuns, todos podem se beneficiar: professores, estudantes e autores interessados na utilização de sua produção e também no processo criativo de desenvolver e adaptar recursos educacionais. E se esses materiais são pagos com recursos públicos, seja pelos programas de incentivo ao livro e à leitura ou por investimento próprio de governos para produzirem materiais, faz ainda mais sentido que sejam públicos, ou seja, de livre acesso e livre adaptação.

Tornar esse novo mundo de fato possível e acessível para todos, abrindo caminhos para mais e mais processos colaborativos, segue sendo o principal objetivo de todo o movimento REA, especialmente na perspectiva de quem atua com formação de educadores. O contexto da cultura digital que temos hoje favorece que as pessoas tenham voz, abre espaços de troca e de aprendizagem infinitos. Assim, uma ideia pode originar outra ideia, uma experiência pode estimular outra, um resultado pode inspirar vários.

\section{Referências}

1. Cetic.br (2015), Pesquisa TIC Educação 2014, disponível em: http://cetic.br/pesquisa/educacao/

2. Brasil. Instituto Educadigital. Projeto REA.br, disponível em: www.rea.net.br

3. Freitas NK, Rodrigues MH. O livro didático ao longo do tempo: a forma do conteúdo. Rev de Investigação em Arte [Internet]. 2008 jul. [citado 27 jan 2016]; 3(1): [cerca de 8 p.]. Disponível em: http://www.ceart.udesc.br/revista dapesquisa/volume3/numero1/plasticas/melissa-neli.pdf

4. ROSSINI, Carolina. GONZALEZ, Cristiana. REA: o debate em política pública e oportunidades para o mercado. In Recursos Educacionais Abertos - Práticas Colaborativas e Políticas Públicas, Casa da Cultura Digital, 2012, disponível em: http://www.artigos.livrorea.net.br/2012/05/rea-o-debate-em-politicapublica-e-as-oportunidades-para-o-mercado/

5. GONSALES, Priscila. Recursos Educacionais Abertos, Formação de Professores e o Desafio de Educar na Cultura Digital. In TIC Educação 2013, Cetic.br disponível em: http://www.cetic.br/media/docs/ publicacoes/2/tic-educacao-2013.pdf

6. Brasil. Recursos Educacionais Abertos. Declaração REA de Paris 2012. [local desconhecido]: Recursos Educacionais Abertos; 2012. [citado 27 jan 2016]. Disponível em: http://rea.net.br/site/declaracao-reade-paris-2012l

7. Open Educational Resources. Initiative Background. Taking OER beyond the OER Community; 2012. [cited 2016 jan 27]. Available: http://oerworkshop.weebly.com/

8. Brasil.Secretaria do Governo Municipal, Camara Municipal de São Paulo. Decreto no 52.681, de 26 de setembro de 2011.Dispõe sobre o licenciamento obrigatório das obras intelectuais produzidas comobjetivos educacionais, pedagógicos e afins, no âmbito da rede pública municipal deensino. [Internet]. Camara Municipal de São Paulo, São Paulo; 26 set 2011. [citado 27 jan 2016]. Disponível em: http://rea.net.br/site/politicas-publicas-para-rea/decreto-municipal-da-cidade-de-sao-paulo/

9. Porvir. Moocs mudam o ensino dentro e fora da universidade. Porvir [Internet]. [citado 27 jan 2016]. Disponível em: http://porvir.org/porpessoas/moocs-mudam-ensino-dentro-fora-dauniversidade/20130404

10. Brasil. Secretaria de Educação do Estado de São Paulo. Currículo+ | Tecnologia a serviço da aprendizagem. [citado 27 jan 2016]. Disponível em: http://curriculomais.educacao.sp.gov.br/ 